\title{
Registros de espécies de mamíferos e aves ameaçadas em Mato Grosso do Sul com ênfase no Sistema Estadual de Unidades de Conservação
}

\author{
Sylvia Torrecilha', Roberto Machado Gonçalves', Rudi Ricardo Laps², \\ Walfrido Moraes Tomas ${ }^{3}$, Helen Liepkan Maranhão² \& Fabio de Oliveira Roque ${ }^{2}$
}

\begin{abstract}
1. Instituto de Meio Ambiente do Mato Grosso do Sul, Campo Grande, MS, Brasil.
2. Centro de Ciências Biológicas e da Saúde, Universidade Federal do Mato Grosso do Sul, Campo Grande, MS, Brasil.

3. Embrapa Pantanal, Corumbá, MS, Brasil.
\end{abstract}

Recebido 7 dezembro 2016

Aceito 6 fevereiro 2017

DOI: $10.1590 / 1678-4766 e 2017156$

ABSTRACT. Records of threatened birds and mammals in Mato Grosso do Sul State focusing on protected areas. We evaluated the records of threatened birds and mammals in protected areas of Mato Grosso do Sul State (Brazil) aiming to build a georeferenced and systematic database to support conservation initiatives. We applied as selection criteria those species occurring in national and global red lists. We recorded 38 bird and 20 mammal species present on the red lists. Our study suggests the need for elaborating strategic plans to conserve threatened species in Mato Grosso do Sul.

KEYWORDS. Threatened species, protected areas, database, Brazil.

RESUMO. Nós avaliamos os registros de espécies ameaçadas de extinção nas unidades de conservação do Estado de Mato Grosso do Sul com o objetivo de construir uma base de dados sistematizada e georreferenciada para auxiliar ações de conservação. Utilizamos como critério de seleção de espécies aquelas presentes na lista nacional e/ou global. Registramos 38 espécies de aves e 20 mamíferos na lista de ameaçados. Em síntese, nosso estudo sugere a necessidade de elaboração de planos de ação para conservação de espécies ameaçadas em Mato Grosso do Sul.

PALAVRAS-CHAVE. Espécies ameaçadas, Unidades de Conservação, banco dados, Brasil.

Áreas protegidas constituem a principal estratégia in situ para conservação da biodiversidade no planeta (RODRIGUES et al., 2004). Atualmente são mais de 150.000 áreas protegidas englobando $13 \%$ da superfície do globo (WDPA, 2012). A despeito da sua importância, elas não representam toda parcela da biodiversidade (WITTEMYER et al., 2008) e há evidências de reduções populacionais de espécies nas áreas já estabelecidas (LAURANCE et al., 2012), o que demanda ações de manejo e fortes investimentos na ampliação e consolidação do sistema.

A Convenção sobre Diversidade Biológica (CDB), promulgada em 1992 é o principal instrumento internacional de colaboração entre as partes contratantes para regular as políticas de conservação da diversidade biológica, a utilização sustentável de seus componentes e a repartição justa e equitativa de seus benefícios (MMA, 2000). Durante a Conferência das Partes (COP) 10, com a assinatura do Protocolo de Nagoya, foram estabelecidas as Metas de Aichi - Plano Estratégico 2010-2020. Dentre os nossos desafios, de metas a atingir até 2020, o país se propõe, através da Meta de Aichi 11, "conservar até 2020 por meio de sistemas de áreas protegidas - ecologicamente representativas, geridas de maneira efetiva e equitativa - pelo menos $17 \%$ de áreas terrestres e 10\% de águas continentais" (WEIGAND JR et al., 2011).

Apesar do Brasil ser responsável por cerca de $75 \%$ das áreas protegidas criadas no mundo desde 2003 (JENKINS \& JOPPA, 2009), há desequilíbrios considerando sistemas territoriais e aqueles delimitados por eco-regiões. Enquanto as Unidades de Conservação (UC's) da Amazônia têm grande abrangência, em outros biomas (ex. Pantanal) a sua extensão é bastante reduzida (WeIGAND JR et al., 2011). Segundo dados do ICMBio (NASCIMENTO \& CAMPOS, 2011), atualmente o país conta com seguinte situação em termos de representatividade de UC's nos sistemas brasileiros: Amazônia (27\%), Mata Atlântica (8,9\%), Cerrado (8,2\%), Caatinga (7,3\%), Pantanal (4,7\%), Pampa (3,5\%) e Ecossistemas Marinhos (1,5\%), sendo $48,15 \%$ sob jurisdição federal e 48,83\% sob jurisdição estadual, $3,02 \%$ sob jurisdição municipal e $0,36 \%$ de natureza privada, totalizando 1.322 unidades de conservação.

No Mato Grosso do Sul (MS) a história da implantação de instrumentos e ferramentas conservacionistas é bastante recente, sendo um dos últimos membros da federação a criar unidades de conservação. O Macrozoneamento Geoambiental do Mato Grosso do Sul (SEPLAN-MS, 1986) constitui-se no primeiro documento público de indicação de uma área para a 
conservação, notadamente as formações cársticas de cobertura florestal da Serra da Bodoquena. No entanto, decorreu mais de uma década até a criação da primeira unidade de conservação de proteção integral no Mato Grosso do Sul, materializada com a criação do Parque Estadual Várzeas do Ivinhema, em dezembro de 1998, com 73.000 hectares, nas formações florestais do domínio da Mata Atlântica e áreas úmidas, criado como compensação ambiental pela construção da UHE Sergio Motta, no rio Paraná. Atualmente, o estado possui 28 unidades de conservação de Proteção Integral abrangendo 320.170,62 hectares que representam $0,9 \%$ da sua superfície territorial. Este sistema é formado por unidades das três esferas de gestão, assim caracterizados: três Parques Nacionais sob jurisdição federal, seis Parques e dois Monumentos Naturais sob jurisdição estadual e 17 Parques e Monumentos Naturais municipais. As Reservas Particulares do Patrimônio Natural (RPPN's) totalizam 12 federais e 35 estaduais, representando 0,39\% da superfície do Mato Grosso do Sul. Portanto, o estado assegura a proteção de $1,29 \%$ da sua superfície com unidades de conservação do grupo de Proteção Integral. Das categorias de Uso Sustentável, o estado possui 39 unidades de conservação (uma federal, duas estaduais e 37 municipais), abrangendo 4.700.125,39 hectares que representam 13,16\% de superfície protegida, predominantemente da categoria Áreas de Proteção Ambiental, na sua grande maioria da esfera municipal.

Embora a superfície total de unidades de conservação do Mato Grosso do Sul não esteja tão aquém da Meta 11 de Aichi para 2010-2020, para sua consolidação e ampliação o sistema precisa integrar dois elementos: representatividade e efetividade. A representatividade biológica das redes de UC's depende não apenas da área total, mas da otimização de conservação de valores (por exemplo, riqueza de espécies, diversidade funcional, endemismos, diversidade filogenética) e objetos (ex. grupos taxonômicos, habitats) que podem ser selecionados através de planejamento sistemático (Loyola et al., 2007). Já a efetividade de conservação da biodiversidade envolve tanto a efetividade de gestão das UC's (ver ERvIN, 2003 Rapid Assessment and Priorization of Protected Area Management/RAPPAM/WWF) como efetividade de conservação da biodiversidade stricto sensu.

A sistematização de dados de biodiversidade que permitam monitoramento de metas é um pré-requisito para consolidação e ampliação do sistema de UC's no estado e melhorias na sua representação e efetividade. Embora o Instituto Chico Mendes de Conservação da Biodiversidade (ICMBio) tenha avançado na direção de organizar e disponibilizar para sociedade dados sobre a biodiversidade nas UC's federais (NASCIMENTO \& CAMPOS, 2011), a maioria dos estados, incluindo o Mato Grosso do Sul, ainda não possui sistemas de monitoramento de biodiversidade em UC's.

Neste artigo, como parte das iniciativas do Biota-MS para atingir as Metas de Aichi 11 (expandir e implementar sistemas de áreas protegidas) e 12 (evitar extinções de espécies), nós avaliamos os registros de espécies de mamíferos e de aves ameaçadas de extinção nas unidades de conservação do Mato Grosso do Sul com o objetivo de construir uma base de dados sistematizada e georreferenciada para subsidiar ações de ampliação e consolidação do sistema de UC's estadual.

\section{MATERIAL E MÉTODOS}

Realizamos um levantamento dos registros de espécies de aves e mamíferos ameaçados no MS. Optamos pela seleção destes grupos taxonômicos, pois (i) são amplamente utilizados como indicadores ambientais, (ii) pela disponibilidade de dados na literatura regional e internacional, (iii) por constituírem grupos com muitas espécies listadas como ameaçadas, (iv) por apresentarem muitas espécies cinegéticas e bandeiras.

Nós utilizamos como critério de seleção de espécies aquelas presentes na lista nacional (IBAMA, 2003) e/ou global (IUCN, 2013) consideradas Ameaçadas (Criticamente em Perigo, Em Perigo e Vulnerável) e Quase Ameaçadas. Para os levantamentos dos registros foram utilizados Machado et al. (2008), Planos de Manejo de Unidades de Conservação, Estudos de Impacto Ambiental (EIA) e Planos de Auto Monitoramento requeridos para monitoramento de empreendimentos disponíveis para acesso público nos setores de licenciamento ambiental do Instituto de Meio Ambiente do Mato Grosso do Sul (IMASUL/MS). Para compilar artigos publicados sobre a fauna do Estado utilizamos o sistema de busca da base de dados ISI Web of Knowledge. Utilizamos as seguintes palavras-chave: Mato Grosso do Sul AND mamíferos OR mammal* OR aves OR bird*.

Para evitar problemas relacionados à desatualização da nomenclatura taxonômica, utilizamos documentos produzidos nos últimos anos (por ex. estudos de impacto ambiental a partir de 2008). Para nomenclatura das aves utilizamos o CBRO (2011), enquanto que para os mamíferos foi utilizada a Lista Anotada de Mamíferos do Brasil (PAGLIA et al., 2012). Foram descartados todos os documentos que não apresentavam confiabilidade de campo (dados de natureza secundária, sem a realização de esforço amostral de campo, inconsistências com a distribuição conhecida da espécie) e ocorrências com localização geográfica imprecisas. Cada registro identificado corresponde à existência de ao menos um registro de ocorrência de uma espécie de aves ou mamíferos ameaçada em uma determinada região. No caso de UC's cuja área também abrange estados vizinhos ao Mato Grosso do Sul (p. ex. Parque Nacional de Emas) foi considerado o registro na UC, independente da localidade.

Para a construção do banco de dados em modelo de SIG foram geradas tabelas específicas das informações de ocorrência das espécies, contendo: número de registros, local encontrado, bacia hidrográfica, bioma, município e classificação da ameaça na legislação nacional e IUCN. Esta tabela contém ainda as coordenadas geográficas padronizadas em graus decimais, as quais foram processadas no software ArcGis (versão 10.0), gerando os shapefiles com todos os registros de ocorrência de cada espécie de aves e mamíferos ameaçados presentes no âmbito estadual.

As fontes de dados cartográficos utilizadas foram as seguintes: limite estadual - IBGE, 2007; hidrografia 
- IBGE, 2001; biomas - IBGE, 2004; limites das UC's federais (base de dados do ICMBio, 2010); limite das unidades de conservação estaduais e municipais (base de dados do IMASUL, 2012). Foram analisadas 28 unidades de conservação de proteção integral, que totalizam 320.170,62 ha; 40 unidades de conservação de uso sustentável, que totalizam 4.700.080,12 hectares e 48 RPPN's, que totalizam 142.679,62 hectares (Tabs I a III). A bibliografia utilizada para compilação dos dados, bem como da base cartográfica, pode ser obtida por solicitação aos autores.

\section{RESULTADOS}

Nós sistematizamos 920 registros de espécies de aves e mamíferos ameaçados no estado (Tabs IV, V). Para o grupo de aves contabilizamos 292 registros de 38 espécies (Tab. IV). Deste total, 34 espécies apresentaram pelo menos um registro no interior das unidades de conservação. Totalizamos 119 registros dentro de UC's, os quais representam 40,75\% dos registros encontrados.

Considerando o papel de cada unidade de conservação para o grupo de aves, temos o seguinte quadro: o Parque Nacional das Emas abriga o maior número de espécies e registros (17 espécies), seguido do Parque Nacional da Bodoquena com nove espécies, o Parque Estadual Pantanal do Rio Negro e Parque Estadual Nascentes do Rio Taquari, ambos abrigando três espécies. No caso das RPPN's, a RPPN Rio Negro apresentou 13 espécies, a RPPN Caiman nove, a RPPN Nhumirim cinco e as RPPN's Cabeceiras Prata e da Barra, três espécies.

Neochen jubata (Spix, 1825) (pato-corredor), Amazona vinacea (Kuhl, 1820) (papagaio-de-peito-roxo), Eleothreptus anomalus (Gould, 1838) (curiango-do-banhado) e Alectrurus risora (Vieillot, 1824) (tesoura-do-campo) não foram registradas em UC's.
Para os mamíferos sistematizamos 628 registros de 20 espécies presentes na lista nacional (IBAMA, 2003) ou global (IUCN, 2013) (Tab. V). Deste total, 19 espécies apresentaram pelo menos um registro no interior de unidades de conservação. Foram totalizados 279 registros no interior de unidades de conservação, que representam 44,4\% do total de registros encontrados. Somente Lonchophylla dekeyseri Taddei et al., 1983 (morceguinho-do-cerrado) não foi registrada em UC's.

O Parque Estadual Nascentes Rio Taquari abriga o maior número de espécies e ocorrências (15 espécies), seguido do Parque Estadual do Pantanal do Rio Negro (14 registros), Parque Nacional das Emas (10 espécies), Parque Nacional da Bodoquena e Parque Estadual Várzeas Ivinhema (oito espécies). Para as RPPN's obtivemos a seguinte ordem decrescente de ocorrências: RPPN's Rio Negro (14 espécies), Nhumirim e Buraco das Araras (sete espécies).

\section{DISCUSSÃO}

A falta de informação sobre onde concentrar esforços de conservação é um obstáculo a ser transposto para a conservação da biodiversidade tropical (LoyolA et al., 2007). Por essa razão, diversas organizações de pesquisa e gestão pública estão sistematizando dados de biodiversidade para subsidiar a elaboração de estratégias de conservação alicerçadas em dados de distribuição, por exemplo, a rede Species link. Em consonância com essas iniciativas, nosso trabalho fornece um checklist e um banco de dados de ocorrências das espécies de aves e mamíferos ameaçados no estado de Mato Grosso do Sul, com especial foco em registros em unidades de conservação.

Do total de 623 aves (NunEs et al., 2017, neste fascículo) e 168 mamíferos registrados para o estado (CÁCEREs et al., 2008; TOMAs et al., 2017, neste fascículo),

Tab. I. Área protegida por Unidades de Conservação de Proteção Integral no Mato Grosso do Sul, Brasil (Fonte: IMASUL, 2013).

\begin{tabular}{lcccc}
\hline Categorias de Unidades de Conservação & Número & $\begin{array}{c}\text { Área } \\
\text { (ha) }\end{array}$ & $\begin{array}{c}\text { Participação Relativa/grupo } \\
(\%)\end{array}$ & $\begin{array}{c}\text { Participação Relativa/Estado } \\
(\%)\end{array}$ \\
\hline Parques Nacionais & 3 & $92.886,60$ & 29,01 & 0,26 \\
Parques e Monumentos Naturais Estaduais & 8 & $190.789,71$ & 59,60 & 0,53 \\
Parques e Monumentos Naturais Municipais & 17 & $36.493,32$ & 11,39 & 0,10 \\
$\quad$ Total de Unidades & 28 & $320.170,62$ & 100,00 & 0,9 \\
\hline
\end{tabular}

Tab. II. Área protegida por Reservas Particulares do Patrimônio Natural (RPPN's) no Mato Grosso do Sul, Brasil (Fonte: IMASUL, 2013).

\begin{tabular}{|c|c|c|c|c|}
\hline & Número & $\begin{array}{l}\text { Área } \\
\text { (ha) }\end{array}$ & $\begin{array}{c}\text { Participação Relativa/grupo } \\
(\%)\end{array}$ & Participação Relativa/Estado (\%) \\
\hline RPPNs Federais & 12 & $81.339,55$ & 57 & 0,22 \\
\hline RPPNs Estaduais & 36 & $61.340,07$ & 43 & 0,17 \\
\hline Total de RPPN's & 48 & $142.679,62$ & 100 & 0,39 \\
\hline
\end{tabular}

Tab. III. Área protegida por Áreas de Proteção Ambiental (APA) no Mato Grosso do Sul, Brasil (Fonte: IMASUL, 2013).

\begin{tabular}{|c|c|c|c|c|}
\hline & Número & $\begin{array}{l}\text { Área } \\
\text { (ha) }\end{array}$ & $\begin{array}{c}\text { Participação Relativa/grupo } \\
(\%)\end{array}$ & Participação Relativa/Estado (\%) \\
\hline APA Federal & 1 & $713.370,43$ & 15,18 & 2,0 \\
\hline APA's Estaduais & 2 & $25.548,50$ & 0,54 & 0,70 \\
\hline APA's Municipais & 37 & $3.961 .161,20$ & 84,28 & 11,09 \\
\hline Total Geral & 40 & $4.700 .080,12$ & 100,00 & 13,16 \\
\hline
\end{tabular}




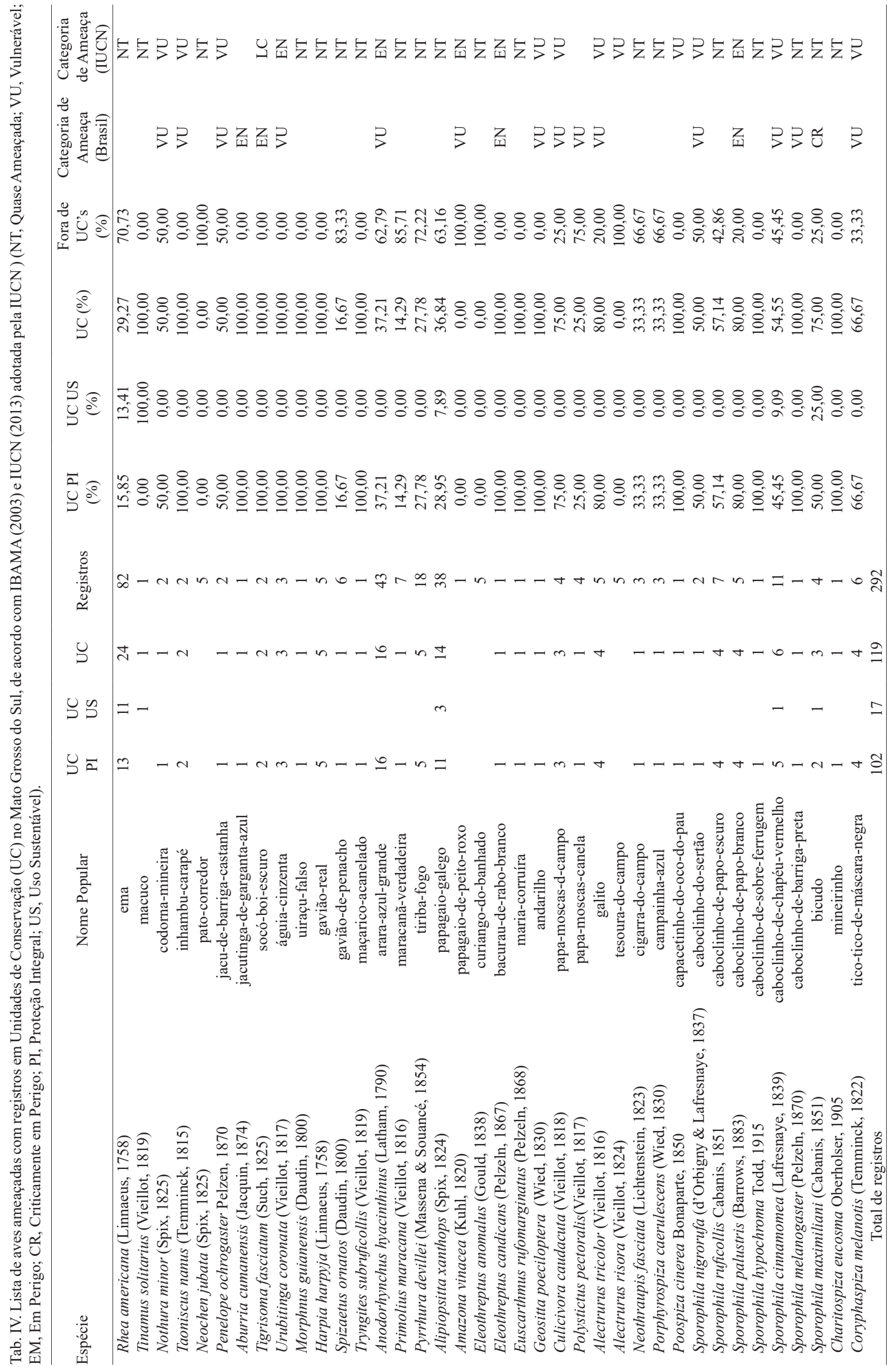







este trabalho registrou 38 espécies de aves e 20 mamíferos na lista de ameaçados. Vale ressaltar que mantivemos em nossa lista cinco espécies de aves registradas apenas na parte goiana do Parque Nacional de Emas para sinalizar a necessidade de se ampliar esforços de amostragem na parte sul-mato-grossense dos parques e áreas adjacentes.

Em relação à abrangência dos estudos no Estado, 62\% das UC's possuem inventários de biodiversidade. Entretanto, a maioria dos estudos apresentam pouca abrangência espacial e continuidade temporal (1 a 5 registros), o que dificulta avaliações de status de conservação das espécies nas áreas. Das unidades amostradas, somente o Parque Nacional das Emas apresenta esforço amostral continuado para alguns grupos. Três unidades de proteção integral e duas de uso sustentável carecem de inventários. Para o grupo de proteção integral destacam-se pela ausência de informações biológicas o Parque Estadual da Serra de Sonora (município de Sonora), o Monumento Natural Estadual do Rio Formoso e o Monumento Natural Estadual Gruta do Lago Azul (ambas no município de Bonito). No caso do grupo de Uso Sustentável destaca-se pela falta de dados a Estrada Parque de Piraputanga. É fundamental a realização de inventários para estas unidades, e implementação de monitoramento sistemático em todas as unidades estaduais, os quais devem estar vinculados aos seus respectivos planos de manejo.

Para alguns grupos, existem avaliações populacionais em determinadas áreas em Mato Grosso do Sul, como cervos (Mourão et al., 2000; Tomas et al., 2004) e queixadas (DesBiez et al., 2009) no Pantanal. Entretanto, não temos informações populacionais para a maioria das espécies ameaçadas nas UC's, com raras exceções, como as estimativas de cervos e veados campeiro no Parque Estadual do Pantanal do Rio Negro (Tomas et al., 2004). Embora os registros sejam o primeiro passo na avaliação do status de conservação das espécies, dados populacionais são particularmente importantes no contexto das análises de viabilidade. A rede de áreas protegidas no estado é significativamente desigual, considerando a porcentagem de unidades de conservação de proteção integral $(1,29 \%)$ comparada com aquelas de uso sustentável (14,45\%), indicando a necessidade reavaliação das estratégias da rede de UC's buscando maior equilíbrio de categorias de manejo para assegurar a proteção da biodiversidade regional. Em síntese, consideramos essencial uma avaliação de viabilidade populacional das espécies ameaçadas, considerando a rede de UC's como parte do plano de ação de conservação dessas espécies em Mato Grosso do Sul.

O mapeamento dos registros de ocorrência de espécies ameaçadas não deve ser visto como uma atividade finalista, mas sim como o primeiro passo para o conhecimento da ocorrência e padrão de distribuição das espécies nas diversas regiões ecológicas e biomas brasileiros, diminuindo os grandes déficits de conhecimento sobre a identidade e distribuição das espécies da região tropical (BINI et al., 2006). Nosso estudo apresenta um panorama abrangente dos registros de espécies ameaçadas nas UC's, mas certamente não engloba muitos registros disponíveis fora de unidades de conservação. A maioria destes dados não contabilizados está em dissertações, teses e trabalhos de pesquisas não publicados que estão sendo incluídos no banco de dados do Programa Biota-MS. Além disso, nosso estudo no nível estadual utilizou as lista de espécies ameaçadas em nível global e nacional, sendo que algumas espécies não incluídas na nossa análise podem estar regionalmente sob um grau de ameaça maior que aquele aqui empregado (ver discussão sobre alinhamento entre listas internacionais e nacionais em BRito et al., 2010). Este é um aspecto preocupante, o qual deverá subsidiar a elaboração de uma lista estadual de espécies ameaçadas do Mato Grosso do Sul como forma de refinar nossa análise e adotar medidas que protejam espécies que estejam ameaçadas no âmbito regional.

Em síntese, os registros de ocorrência são essenciais para elaboração de instrumentos de gestão da política nacional de conservação da biodiversidade, com promulgação da Convenção da Biodiversidade (Eco/92). Entendemos que a organização e sistematização de dados de ocorrência das espécies ameaçadas em Sistema de Informação Geográfica é a base para elaboração de estratégias de conservação como planejamento sistemático para conservação, elaboração de listas de espécies ameaçadas, planos de conservação, priorização e incremento da rede de unidades de conservação e avaliações de eficiência de gestão previstas para serem elaboradas no estado nos próximos anos.

Agradecimentos. À Fundação de Apoio ao Desenvolvimento do Ensino, Ciências e Tecnologia do Estado de Mato Grosso do Sul (Fundect) e a Superintendência de Ciências e Tecnologia do Estado de Mato Grosso do Sul (Sucitec/MS) pelo convite de participação neste fascículo especial da Iheringia, Série Zoologia e o suporte financeiro para sua publicação. Ao técnico do NEMAE/IMASUL João Paulo Rosa dos Santos pelo apoio na construção do banco de dados. Ao acadêmico de biologia da UFMS Henrique Takashi Mitsuyasu no apoio à elaboração dos mapas.

\section{REFERÊNCIAS BIBLIOGRÁFICAS}

Bini, L. M.; Diniz-Filho, J. A. F.; Rangel, T. F. L. V. B.; Bastos, R. P. \& PINTO, M. P. 2006. Challenging Wallacean and Linnean shortfalls: knowledge gradients and conservation planning in a biodiversity hotspot. Diversity and Distributions 12:475-482.

Brito, D.; Ambal, R. G.; Brooks, T.; Silva, N. D.; Foster, M.; Hao, W.; Hilton, T. C.; Paglia, A.; Rodríguez, J. P. \& Rodríguez, J. V. 2010. How similar are national red lists and the IUCN Red List? Biological Conservation 143:1154-1158.

Cáceres, N. C.; Carmignotto, A. P.; Fischer, E. A. \& Santos, C. F. 2008. Mammals from Mato Grosso do Sul, Brazil. CheckList 4(3):321-335.

CBRO - Comitê Brasileiro de Registros Ornitológicos. 2011. Listas das aves do Brasil. 10ed, 25/1/2011. Disponível em: $<\mathrm{http}: / / w w w$. cbro.org.br>. Acessado em 20.7.2013.

Desbiez, A. L. J.; Bodmer, R. E. \& Tomas, W. M. 2009. Mammalian densities in a Neotropical wetland subject to extreme climatic events. Biotropica 42:372-378

ERVIN, J. 2003. Metodologia para avaliação rápida e priorização do manejo de unidades de conservação (RAPPAM). Gland, WWF. 70p.

IBAMA. 2003. Lista das espécies da fauna brasileira ameaçadas de extinção. Anexo à Instrução Normativa n ${ }^{\circ}$ 3, de 27 de maio de 2003. Brasília, Ministério do Meio Ambiente.

ICMBio - Instituto Chico Mendes de Conservação da Biodiversidade. 2010. Parques Nacionais. Disponível em: $<\mathrm{http}: / / \mathrm{www}$.icmbio.gov. br/portal/>. Acessado em: 23.10.2010.

imaSUl - Instituto de Meio Ambiente de Mato Grosso do Sul. 2012. Disponível em: <http://www.imasul.ms.gov.br/>. Acessado em 20.11.2012. 
IUCN. 2013. IUCN Red list of threatened species. Version 2013.1. Disponível em: < www.iucnredlist.org $>$. Acessado em 17.07.2013.

JENKINS, C. N. \& JoPPA, L. 2009. Expansion of the global terrestrial protected area system. Biological Conservation 142:2166-2174.

Laurance, W. F.; Useche, D. C.; Rendeiro, J.; Kalka, M. B.; BradshaW, C. J. A.; Sloan, S. P.; Laurance, S. G.; Campbell, M.; Abernethy, K.; Alvarez, P.; Arroyo-Rodriguez, V.; Ashton, P.; Benítez-MY, J.; Blom, A.; Bobo, K. S.; Cannon, C. H.; CaO, M.; Carroll, R.; Chapman, C.; Coates, R.; Cords, M.; Danielsen, F.; Dijn, B. D.; Dinerstein, E.; Donnelly, M. A.; Edwards, D.; Edwards, F.; FarwiG, N.; Fashing, P.; Forget, P. -M.; Foster, M.; Gale, G.; Harris, D.; Harrison, R.; Hart, J.; Karpanty, S.; Kress, W. J.; Krishnaswamy, J.; Logsdon, W.; Lovett, J.; Magnusson, W.; Maisels, F.; Marshall, A. R.; McClearn, D.; Mudappa, D.; Nielsen, M. R.; Pearson, R.; Pitman, N.; Ploeg, J. V. D.; Plumptre, A.; Poulsen, J.; Quesada, M.; Rainey, H.; Robinson, D.; Roetgers, C.; Rovero, F.; Scatena, F.; Schulze, C.; Sheil, D.; Struhsaker, T.; Terborgh, J.; Thomas, D.; Timm, R.; Urbina-Cardona, J. N.; Vasudevan, K.; Wright, S. J.; AriaS-G., J. C.; Arroyo, L.; Ashton, M.; AuZel, P.; BabaASA, D.; BabWeteera, F.; BaKer, P.; Banki, O.; Bass, M.; Bila-Isia, I.; Blake, S.; Brockelman, W.; BroKaW, N.; Brühl, C. A.; Bunyavejchewin, S.; Chao, J.-T.; Chave, J.; Chellam, R.; Clark, C. J.; Clavijo, J.; Congdon, R.; Corlett, R.; Dattaraja, H. S.; Dave, C.; Davies, G.; Beisiegel, B. M.; Silva, R. N. P.; Fiore, A. D.; Diesmos, A.; Dirzo, R.; Doran-Sheehy, D.; Eaton, M.; Emmons, L.; Estrada, A.; Ewango, C.; Fedigan, L.; Feer, F.; Fruth, B.; Willis, J. G.; Goodale, U.; Goodman, S.; Guix, J. C.; Guthiga, P.; Haber, W.; Hamer, K.; Herbinger, I.; Hill, J.; HuAnG, Z.; Sun, I. F.; ICKes, K.; ItoH, A.; Ivanauskas, N.; Jackes, B.; JANOVEe, J.; JanZen, D.; JiAngming, M.; Jin, C.; Jones, T.; Justiniano, H.; Kalko, E.; Kasangaki, A.; Killeen, T.; King, H.-B.; Klop, E.; Knott, C.; Koné, I.; Kudavidanage, E.; Ribeiro, J. L. S.; LattKe, J.; Laval, R.; Lawton, R.; Leal, M.;. Leighton, M.; Lentino, M.; Leonel, C.; Lindsell, J.; Ling-Ling, L.; Linsenmair, K. E.; Losos, E.; Lugo, A.; Lwanga, J.; Mack, A. L.; Martins, M.; McGraw, W. S.; McNab, R.; MontaG, L.; Thompson, J. M.; Nabe-Nielsen, J.; NaKagaWa, M.; Nepal, S.; NorConK, M.; Novotny, V.; O’Donnell, S.; Opiang, M.; Ouboter, P.; Parker, K.; Parthasarathy, N.; Pisciotta, K.; Prawiradilaga, D.; Pringle, C.; Rajathurai, S.; Reichard, U.; Reinartz, G.; Renton, K.; Reynolds, G.; Reynolds, V.; Riley, E.; RöDel, M.-O.; Rothman, J.; Round, P.; SaKaI, S.; Sanaiotti, T.; SaVini, T.; SchaAb, G.; SeIdensticker, J.; Siaka, A.; Silman, M. R.; Smith, T. B.; Almeida, S. S.; Sodhi, N.; Stanford, C.; Stewart, K.; Stokes, E.; Stoner, K. E.; Sukumar, R.; Surbeck, M.; Tobler, M.; TscharntKe, T.; Turkalo, A.; Umapathy, G.; Weerd, M. V.; Rivera, J. V.; Venkataraman, M.; Venn, L.; Verea, C.; Castilho, C. V.; Waltert, M.; Wang, B.; Watts, D.; Weber, W.; West, P.; Whitacre, D.; Whitney, K.; Wilkie, D.; Williams, S.; Wright, D.; Wright, P.; Xiankai, L.; Yonzon, P. \& Zamzani, F. 2012. Averting biodiversity collapse in tropical forest protected areas. Nature 489:290-294.

Loyola, R. D.; KuBOtA, U. \& Lewinsohn, T. M. 2007. Endemic vertebrates are the most effective surrogate for identifying conservation priorities among Brazilian ecoregions. Diversity and Distributions 13:389-396.
Machado, A. B. M.; Drummond, G. M. \& Paglia, A. P. eds. 2008 Livro vermelho da fauna brasileira ameaçada de extinção. (Série Biodiversidade, 19). Brasília, Ministério do Meio Ambiente, 2v. 1420p.

Ministério do Meio Ambiente. 2000. A Convenção sobre Diversidade Biológica - CDB. Brasília, Ministério do Meio Ambiente, Secretaria de Biodiversidade e Florestas. 30p.

Mourão, G.; Coutinho, M. E.; Mauro, R. A.; Campos, Z.; Tomás, W. \& Magnusson, W. 2000. Aerial surveys of caiman, marsh deer and pampas deer in the Pantanal wetland of Brazil. Biological Conservation 92:175-183.

NASCimento, J. L. \& CAmpos, I. B. eds. 2011. Atlas da fauna brasileira ameaçada de extinção em unidades de conservação federais. Brasília, Instituto Chico Mendes de Conservação da Biodiversidade (ICMBio). 276p.

Nunes, A. P.; Straube, F. C.; LaPs, R. R. \& Posso, S. R. 2015. Checklist das aves do Estado do Mato Grosso do Sul, Brasil. Iheringia, Série Zoologia (neste Volume).

Paglia, A. P.; Fonseca, G. A. B.; Rylands, A. B.; Herrmann, G.; Aguiar, L. M. S.; Chiarello, A. G.; Leite, Y. L. R.; Costa, L. P.; Siciliano, S.; Kierulff, M. C. M.; Mendes, S. L.; Tavares, V. C.; MitTermeier, R. A. \& PATtON, J. L. 2012. Lista anotada dos mamíferos do Brasil. 2ed. Arlington, Conservation International. 76p.

Rodrigues, A.; Rodrigues, A. S. L.; ANDElman, S. J.; BAKARR, M. I.; Luigi, B.; Brooks, T. M.; Cowling, R. M.; Fishrool, L. D. C.; FonseCA, G. A. B.; Gaston, K. J.; Hoffmann, M.; Long, J. S.; Marquet, P. A.; Pilgrim, J. D.; Pressey, R. L.; Schipper, J.; Sechrest, W.; Stuart, S. N.; UNDERHILl, L. G.; WALLER, R. W.; WATTS, M. E. J. \& YAN, X. 2004. Effectiveness of the global protected area network in representing species diversity. Nature 428:640-642.

SEPLAN-MATO GROSSO DO SUL. 1986. Macrozoneamento geoambiental do Mato Grosso do Sul. Campo Grande, Secretaria de Planejamento.

Tomas, W. M.; Zucco, C. A.; Fernandez, F. A.; Harris, M.; Cardim, E. N.; Cestari, C.; Costa, R. L.; Ferreira, V. L.; Hulle, N. L.; Indrusiak, C. B.; Kalerhoff, M.; Medeiros, T. T.; Pinheiro, A. R. T.; Rimoli, J.; Santos, A.; Santos, J. R. N.; TAPia, G. L. G. \& Michelson, M. A. T. 2004. Estimativa da abundância das populações de cervo (Blastocerus dichotomus) e veado campeiro (Ozotoceros bezoarticus) no Parque Estadual do Pantanal do Rio Negro, MS. In: IV Simpósio sobre Recursos Naturais e Sócio-Econômicos do Pantanal. Corumbá, Embrapa. 109p.

Tomas, W. M.; Antunes, P. M.; Bordignon, M. O.; Camilo, A. R.; Campos, Z.; Camargo, G.; Carvalho, L. F. A. C.; Cunha, N. L.; Fischer, E.;Godoi, M. N.; Hannibal, W.; Mourão, G.; Rimoli, J.; Santos, C. F.; Silveira, M. \& Tomas, M. A. 2017. Checklist of mammal species from Mato Grosso do Sul, Brazil. Iheringia, Série Zoologia, 107(supl.): e2017155

WDPA. World Database on Protected Areas. Disponível em: <www.wdpa. org $>$. Acessado em 01.04.2013.

Weigand JR., R.; Silva, D. C. \& Silva, D. O. 2011. Metas de Aichi. Situação atual no Brasil. Brasília, IUCN, WWF-Brasil, Ipê. 67p.

Wittemyer, G.; Elsen, P.; Bean, W. T.; Burton, A. C. O. \& Brashares, J. S. 2008. Accelerated human population growth at protected area edges. Science 321:123-126. 